\title{
Micromagnetic Simulations of Magnetization Reversals in Nd-Fe-B Based Permanent Magnets
}

\author{
H. Sepehri-Amin*, T. Ohkubo and K. Hono \\ Elements Strategy Initiative Center for Magnetic Materials (ESICMM), National Institute for Materials Science, Tsukuba 305-0047, Japan
}

Finite element micromagnetic simulation was employed to explain how the microstructure of Nd-Fe-B permanent magnets such as grain size, grain shape, and grain boundary composition influence the magnetization reversals and coercivity. Micromagnetic simulations showed that local demagnetization factor decreases as grain size decreases, which is attributed to a higher coercivity in fine-grained anisotropic permanent magnets. Lower demagnetization factor is also responsible for a lower temperature dependence of coercivity in the magnets with a smaller grain size. It was also found that the reduction of the magnetization of the grain boundary phase in hot-deformed Nd-Fe-B magnets leads to the coercivity enhancement due to a stronger pinning force against magnetic domain wall motion. The coercivity of Nd-Fe-B magnets cannot be enhanced by the reduction of the grain size alone unless the grain boundary phase become non-ferromagnetic, indicating that the role of the grain boundary phase is more pronounced in the Nd-Fe-B magnets with a smaller grain size. [doi:10.2320/matertrans.M2015457]

(Received December 8, 2015; Accepted May 10, 2016; Published June 24, 2016)

Keywords: micromagnetic simulations, neodymium-iron-boron magnets, coercivity, and demagnetization field

\section{Introduction}

The development of high coercivity Nd-Fe-B based permanent magnets without use of heavy rare earth elements for traction motor of hybrid/electric vehicles and generator of direct drive wind turbines is the center of research interest in the permanent magnet community ${ }^{1)}$. Coercivity, as an extrinsic magnetic property, can be controlled by microstructure of the magnet such as grain size, grain shape, grain boundary and interphase interfaces. In order to understand the reasons for the low coercivity of Nd-Fe-B based commercial permanent magnets, multi-scale microstructure characterizations have been employed ${ }^{2)}$. However, microstructure observation by itself cannot explain the underlying physics for the unexpectedly low coercivity. Computational simulation is a strong tool that can give a better understating on the magnetization reversal process of $\mathrm{Nd}-\mathrm{Fe}-\mathrm{B}$ magnets with complex microstructures. In this paper, we will review how the micromagnetic simulations can be used to correlate the microstructural features of Nd-Fe-B based permanent magnets to their coercivity.

In micromagnetic simulations, the first step is to make a model close to the microstructure of real magnets. For example, polyhedral shaped grains for $\mathrm{Nd}-\mathrm{Fe}-\mathrm{B}$ sintered magnets, platelet-like shaped grains for hot-deformed magnets, or thin films with columnar grains can be modeled as shown in Fig. 1. Thereafter, the magnetization distribution in the model is resolved by subdividing the grains to small triangles, tetrahedrons, with a size much smaller than the grain size. In fact, the size of tetrahedrons in the micromagnetic simulation should be smaller than the exchange length of the ferromagnet $^{3)}$. Constant magnetization modulus can be distributed among each node of the tetrahedrons with the equation of " $\boldsymbol{M}(\boldsymbol{r})=M_{s} \boldsymbol{m}(\boldsymbol{r})$ " in which $\mathbf{m}(\mathrm{r})$ is unit vector in each node. The direction of magnetization in each node can be determined by minimizing the total Gibbs' free energy of system ${ }^{4)}$. The hysteresis loop of the ferromagnetic materials can be cal-

*Corresponding author, E-mail: h.sepehriamin@nims.go.jp culated by minimizing the total Gibbs' free energy at different applied magnetic fields ${ }^{5}$. On the other hand, dynamic response of the magnetization can be calculated at various applied external magnetic fields by solving Landau-Lifshitz-Gilbert (LLG) equation of motion for the magnetization in a dynamic magnetic field ${ }^{6}$.

Total magnetic Gibbs' free energy, $E_{t}$, can be expressed as:

$$
E_{t}=E_{e x}+E_{K}+E_{s}+E_{H}
$$

that is the sum of the exchange energy $\left(E_{e x}\right)$, the magnetocrystalline anisotropy energy $\left(E_{K}\right)$, the stray field energy $\left(E_{s}\right)$, and the Zeeman energy $\left(E_{H}\right)^{4)}$. Considering the external magnetic field as $H_{e x t}$, the total magnetic Gibbs' free energy can be written as:

$$
\begin{aligned}
E_{t}\left(\boldsymbol{M}, \boldsymbol{H}_{\text {ext }}\right)= & \int\left[\frac{A}{M_{S}^{2}} \sum_{k=1}^{3}(\nabla M k)^{2}-\frac{\mu_{0}}{2} \boldsymbol{H}_{\boldsymbol{d}} \cdot \boldsymbol{M}\right. \\
& \left.+f_{k}(\boldsymbol{M})-\mu_{0} \boldsymbol{H}_{\text {ext }} \cdot \boldsymbol{M}\right] d V .
\end{aligned}
$$

in which the effective field is the negative derivative of above equation with respect to the magnetization. In the other words, the effective field is deduced from the variation of total Gibbs' free energy.

$$
\boldsymbol{H}_{\text {eff }}=-\frac{\partial E_{t}}{\partial \boldsymbol{m}}
$$

It can be seen that the total magnetic Gibbs' free energy $\left(E_{t}\right)$ is a function of external magnetic field and magnetization. In this equation, $M_{s}$ is spontaneous magnetization and $M_{k}$ is the kth component of the magnetization vector $\boldsymbol{M}$. $A$ is the exchange constant and $f_{K}$ is the magnetocrystalline energy density, and $\boldsymbol{H}_{\boldsymbol{d}}$ is demagnetization field.

Using Gilbert equation of motions shown below,

$$
\frac{\partial \boldsymbol{m}}{\partial t}=-\frac{\gamma}{1+\alpha^{2}}\left(\boldsymbol{m} \times \boldsymbol{H}_{e f f}\right)-\frac{\alpha}{M_{s}\left(1+\alpha^{2}\right)}\left[\boldsymbol{m} \times\left(\boldsymbol{m} \times \boldsymbol{H}_{e f f}\right)\right]
$$

the time evolution of magnetization can be computed. In the above equation, $\gamma$ is gyromagnetic ratio and $\alpha$ is dimensionless damping factor ${ }^{7}$. Using this equation, precession of 

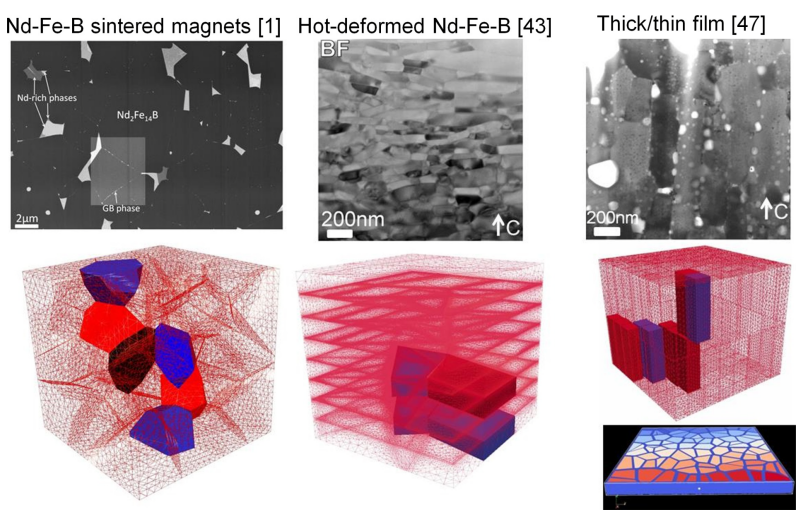

Fig. 1 Development of models for the micromagnetic simulation based on the microstructure of Nd-Fe-B permanent magnets. Reprinted with permission from Elsevier ${ }^{1,43,47)}$.

magnetization in an effective magnetic field considering a viscous damping can be calculated, and the magnetization curve of the model can be simulated by changing external magnetic field dynamically ${ }^{6,7)}$.

\section{Effect of Grain Size on Coercivity and Thermal Sta- bility of Coercivity in Nd-Fe-B Permanent Magnets}

It is known experimentally that the coercivity of $\mathrm{Nd}-\mathrm{Fe}-\mathrm{B}$ permanent magnets increases by the reduction of the grain size following $H_{\mathrm{c}}=a-b \ln D^{8-10)}$. However, by the reduction of grain size below $3 \mu \mathrm{m}$, this relationship fails due to the oxidation of Nd-rich phases in fine-grained sintered magnets ${ }^{11)}$. Une and Sagawa demonstrated a high coercivity of $2 \mathrm{~T}$ using ultrafine-grained $\mathrm{Nd}-\mathrm{Fe}-\mathrm{B}$ sintered magnet $(D \sim 1 \mu \mathrm{m})$ that was processed by controlling the oxygen atmosphere rigorously using the press-less sintering route ${ }^{12)}$. In addition, Liu et al. has shown experimentally that the thermal stability of coercivity can be improved when the grain size of $\mathrm{Nd}-\mathrm{Fe}-\mathrm{B}$ magnet decreases ${ }^{13)}$. Then a question arises: what is the underlying physics on the coercivity enhancement mechanism and improvement of thermal stability of coercivity by the grain size reduction?

Ramesh et al. carried out systematic studies on the correlation between the coercivity and grain size of sintered magnet and proposed that the coercivity increase by the reduction of the grain size is due to the decrease in the defect density on the surface region ${ }^{8)}$. In this hypothesis, it was assumed that magnetic reversal occurs by the nucleation of reverse domains in magnetically isolated grains ${ }^{8}$. However, recent detailed microstructural characterization of $\mathrm{Nd}-\mathrm{Fe}-\mathrm{B}$ sintered magnets has shown that the intergranular phase of sintered magnets is ferromagnetic, so the magnetic grains are exchange coupled ${ }^{2,14,15)}$. In order to realize the underlying physics of the grain size dependency of coercivity, micromagnetic simulations have been employed by several authors ${ }^{16-23}$.

Micromagnetic simulations reproduced the experimental observations of the grain size dependence of coercivity in both isotropic $^{16)}$ and anisotropic ${ }^{19-21)} \mathrm{Nd}-\mathrm{Fe}-\mathrm{B}$ magnets. Using micromagnetic simulations of a $2 \mathrm{D}$ magnetic structure, Schrefl et al. showed that the $\mathrm{Nd}_{2} \mathrm{Fe}_{14} \mathrm{~B}$ grains are strongly coupled by long range magnetostatic interactions, which become less effective by decrease of the grain size, leading to

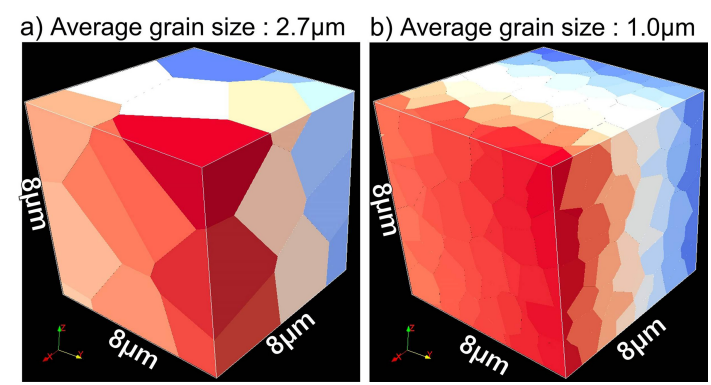

Fig. 2 Modeled sintered magnets with average grain size of (a) $2.7 \mu \mathrm{m}$ and (b) $1.0 \mu \mathrm{m}$. Reprinted with permission from Elsevier ${ }^{23}$.
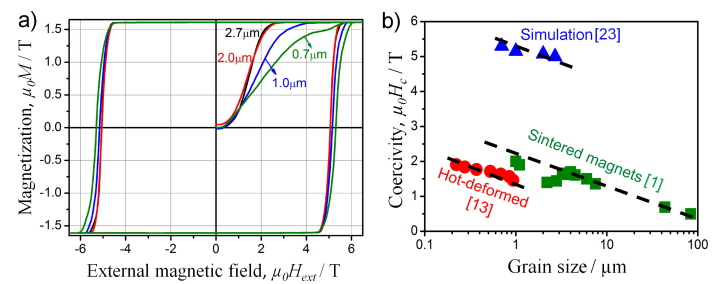

Fig. 3 (a) Magnetization curves of the simulated magnets with average grain sizes of 2.7, 2.0, 1.0, and $0.7 \mu \mathrm{m}$. (b) Comparison of the coercivity versus grain size obtained using micromagnetic simulations and experimentally reported Nd-Fe-B sintered magnets and hot-deformed Nd-Fe-B magnets. Reprinted with permission from Elsevier ${ }^{23)}$.

the decrease of demagnetization field ${ }^{19)}$. The coercivity of hard magnets can be phenomenologically expressed by $H_{\mathrm{c}}=$ $\alpha H_{\mathrm{A}}-N_{\text {eff }} M_{\mathrm{s}}$, where $H_{\mathrm{A}}$ is the anisotropy field of a hard magnetic phase, $N_{\text {eff }}$ is the effective demagnetization factor and $M_{\mathrm{S}}$ is the saturation magnetization. The reduction of the demagnetization field means the reduction of the 2 nd term in this equation leading to the enhancement of coercivity. Kronmüller et al. showed that large coercivity requires exchange decoupling between the grains, and long-range dipolar magnetic stray fields reduces coercivity of the magnets with larger grain size ${ }^{16)}$. Bance et al. simulated grain size dependency of coercivity in a single grained model and explained that logarithmic decay of coercivity with increasing of the grain size results from logarithmic increase in the demagnetizing field near the edge of a grain ${ }^{22)}$. Recently, Sepehri-Amin et al. used finite element micromagnetic simulations of poly crystalline model magnets to understand the underlying mechanism of the grain size dependence of the coercivity in the exchange coupled anisotropic Nd-Fe-B sintered magnets ${ }^{23)}$.

Figure 2 shows the modeled geometries of polyhedron grains with average grain size of 2.7 and $1.0 \mu \mathrm{m}$. In fact the grain size of the models was varied from 2.7 to $0.7 \mu \mathrm{m}$. Sepehri-Amin et al. simulated weakly exchange coupled $\mathrm{Nd}_{2} \mathrm{Fe}_{14} \mathrm{~B}$ grains by assuming $2 \%$ exchange coupling strength of $\mathrm{Nd}_{2} \mathrm{Fe}_{14} \mathrm{~B}$ phase across grain boundaries ${ }^{23}$. Figure 3 (a) shows the simulated hysteresis curves of these models. Coercivity increases by $0.4 \mathrm{~T}$ when the grain size is decreased from $2.7 \mu \mathrm{m}$ to $0.7 \mu \mathrm{m}$ in the exchange coupled models. This figure also demonstrates a decrease in maximum susceptibility as the grain size is reduced. For grain sizes larger than $1 \mu \mathrm{m}$, the magnetization reaches saturation in one step. However, the initial magnetization proceeds in two steps for the model with 1 and $0.7 \mu \mathrm{m}$ grain size. Experimental results also showed a two-step initial magnetization curve when the grain 
a)

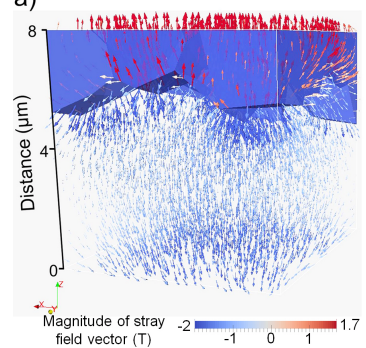

b)

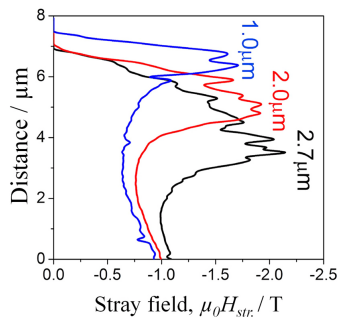

Fig. 4 (a) Distribution of demagnetization vectors in the $2.0 \mu \mathrm{m}$ grain sized model. Note that only surface grains have magnetization values of $\mathrm{M}_{\mathrm{z}}$ / $\mathrm{M}_{\mathrm{s}}=-1$. (b) The distribution of the maximum stray field calculated from surface slices from $Z=0 \mu \mathrm{m}$ to $\mathrm{Z}=8 \mu \mathrm{m}$ for models with grain sizes of $1.0,2.0$, and $2.7 \mu \mathrm{m}$. Reprinted with permission from Elsevier ${ }^{23)}$

size of Nd-Fe-B sintered magnets is reduced to $1 \mu \mathrm{m}^{12,24)}$. The micromagnetic simulation results suggested that the initial high susceptibility is due to the easy displacement of the magnetic domain walls within the multi-domain $\mathrm{Nd}_{2} \mathrm{Fe}_{14} \mathrm{~B}$ grains $^{23)}$. On the other hand, reversal of the magnetization of the single domain particles required higher magnetic fields due to the pinning of the domain walls in the $\sim 5 \mathrm{~nm}$ thick ferromagnetic grain boundary phase. This leads to the twostep magnetization process in the initial magnetization curve of the fine grained Nd-Fe-B sintered magnets ${ }^{23)}$. The coercivity as a function of grain size is shown in Fig. 3 (b) and is compared with that of the experimental results of sintered magnets and hot-deformed Nd-Fe-B magnets ${ }^{1,13,23)}$. The grain size dependence of the coercivity in the simulation follows a similar trend as the experimental observations. However, the unrealistically high coercivity in the simulation is mainly due to the ignorance of the defects that reduce the anisotropy field at surfaces and grain boundaries in real magnets. This discrepancy was recently overcome by introducing a nucleus at the surface of the polycrystalline model ${ }^{25)}$. In addition, thermal fluctuations can further reduce the coercivity, $\sim 15 \%$ for room temperature calculations as reported by Bance et al. ${ }^{26,27)}$, which was not taken into account in this simulation by Sepehri-Amin et al. ${ }^{23)}$.

In the $\mathrm{Nd}-\mathrm{Fe}-\mathrm{B}$ sintered magnets, the surface grains have almost no coercivity due to the defects created during surface machining/polishing or surface oxidation and the magnetization of the surface grains reverse at a lower magnetic field than the nucleation field ${ }^{28)}$. When the magnetization of the surface grains is reversed, the magnetically reversed surface grains cause stray fields to the underlying grains. Sepehri-Amin et al. calculated the stray field generated from the reversed surface grains for different grain sizes as shown in Fig. $4^{23)}$. Figure 4 (a) shows the distribution of demagnetization vectors of $2.0 \mu \mathrm{m}$ grain sized magnet in which the magnetically reversed surface grains are also shown. The arrows show the magnitude and direction of the stray field from the reversed grains. Figure 4 (b) shows the distribution of the maximum stray field calculated from surface slices at $\mathrm{Z}=$ $0 \mu \mathrm{m}$ to $\mathrm{Z}=8 \mu \mathrm{m}$. The largest stray field was found at the interface of the reversed surface grains and the largest value decreases as the grain size decreases. Larger stray fields can induce magnetization reversals of the neighboring grains at lower external magnetic fields in the anisotropic Nd-Fe-B
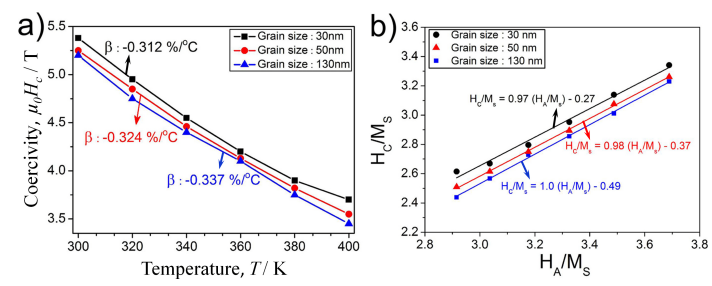

Fig. 5 (a) Coercivity versus temperature of modeled Nd-Fe-B magnets with average grain sizes of 30,50, and $130 \mathrm{~nm}$. (b) The dependence of $H_{\mathrm{c}} / M_{\mathrm{s}}$ versus $H_{\mathrm{A}} / M_{\mathrm{s}}$ of the modeled samples with average grain sizes of 30,50 , and $130 \mathrm{~nm}$. The micromagnetic parameters of $\alpha$ and $N_{\text {eff }}$ are measured from the slope and intercept, respectively, of a linear fit to this graph. Reprinted with permission from Elsevier ${ }^{23)}$.

sintered magnets. Hence, a smaller stray field is beneficial for a higher coercivity, which explains the grain size dependence of coercivity.

The second question is why the thermal stability of coercivity is improved by reducing grain size? The thermal stability of coercivity, $\beta=\left[\Delta H_{c} /\left(H_{c} \times \Delta T\right)\right] \times 100$, is typically $\sim-0.6 \% \mathrm{C}^{-1}$ for commercial Nd-Fe-B sintered magnets without Dy. To enable the magnet to be used at elevated temperature, a low absolute value of $\beta$ is desirable. Liu et al. reported that $\beta$ value improves to the range of $\sim-0.4-0.5 \%{ }^{\circ} \mathrm{C}^{-1}$ by the reduction of grain size to $\sim 250-500 \mathrm{~nm}$ in hot-deformed NdFe-B magnets ${ }^{13)}$. Sepehri-Amin et al. employed micromagnetic simulations to understand the underlying physics of the grain size dependence of $\beta$. A model size of $400 \times 400 \times$ $400 \mathrm{~nm}^{3}$ was constructed with polyhedron shaped grains having average sizes of 30,50 , and $130 \mathrm{~nm}$, which are two orders of magnitude smaller than that of the commercial sintered magnets ${ }^{23}$. Figure 5 (a) shows the simulated coercivity versus temperature for Nd-Fe-B models with grain sizes of 30 , 50 , and $130 \mathrm{~nm}$ and $\beta$ was calculated for these models. The absolute value of $\beta$ increases from $0.312 \% /{ }^{\circ} \mathrm{C}$ for a grain size of $30 \mathrm{~nm}$ to $0.337 \% /{ }^{\circ} \mathrm{C}$ for a grain size of $130 \mathrm{~nm}$. This result suggests that the temperature coefficient of coercivity deteriorates with increase of the grain size, which is in agreement with experimental observations ${ }^{13,23)}$.

Based on the nucleation model, the coercivity of Nd-Fe-B permanent magnets is expressed as:

$$
H_{c}[\mathrm{~T}]=\alpha H_{a}[\mathrm{~T}]-N_{e f f} M_{s}[\mathrm{~T}]
$$

The first term in the above equation corresponds to the influence of microstructural defects on the anisotropy field and the second term is the microstructure-sensitive effective demagnetization ${ }^{29}$. Figure $5(\mathrm{~b})$ shows the $H_{\mathrm{c}} / M_{\mathrm{s}}$ versus $H_{\mathrm{A}} / M_{\mathrm{s}}$ of the modeled Nd-Fe-B magnets with average grain sizes of 30 , 50 , and $130 \mathrm{~nm}$ at different temperatures. A linear fit is applied to this data and the values of $\alpha$ and $N_{\text {eff }}$ are determined. The slopes of the plots do not change much and are nearly 1 since the models does not contain any defects. However, the effective demagnetization constant, $N_{\text {eff }}$, increases from 0.27 for a grain size of $30 \mathrm{~nm}$ to 0.49 for a grain size of $130 \mathrm{~nm}$. This result suggests that the effective local demagnetization factor increases as the grain size increases. This micromagnetic simulation result clearly indicates that the $N_{\text {eff }}$ value increases with grain size.

Both the anisotropy field, $H_{\mathrm{A}}$, and the saturation magnetization, $M_{\mathrm{s}}$, of the $\mathrm{Nd}_{2} \mathrm{Fe}_{14} \mathrm{~B}$ phase decreases as temperature 


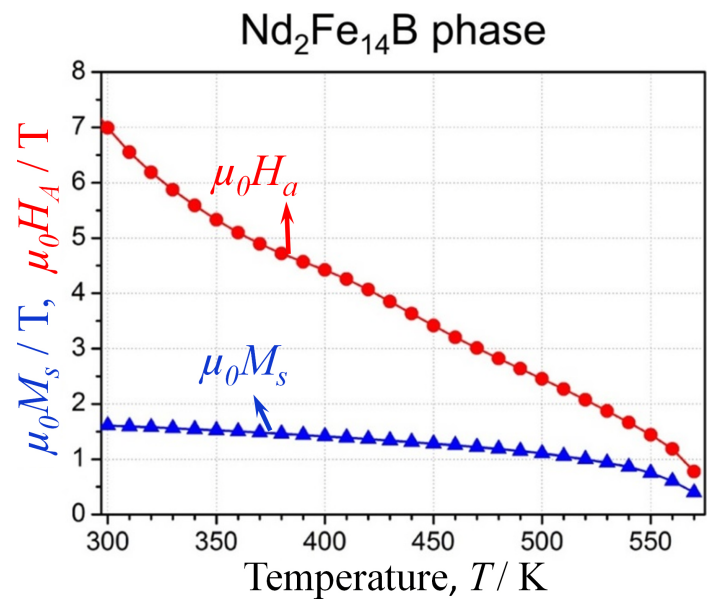

Fig. 6 Saturation magnetization $\left(\mu_{0} M_{\mathrm{s}}\right)$ and magnetic anisotropy field $\left(\mu_{0} H_{\mathrm{A}}\right)$ of $\mathrm{Nd}_{2} \mathrm{Fe}_{14} \mathrm{~B}$ phase measured from room temperature to $570 \mathrm{~K}$ for $\mathrm{Nd}_{2} \mathrm{Fe}_{14} \mathrm{~B}$ phase. The data was measured by Hock ${ }^{30)}$.

increases as shown in Fig. $6^{30,31)}$. However, $H_{\mathrm{A}}$ and $M_{\mathrm{s}}$ do not decrease at the same rate. The anisotropy field decreases from room temperature to $\sim 200^{\circ} \mathrm{C}$ with a sharp negative slope. However, the saturation magnetization decreases at a much slower rate from room temperature to $\sim 160^{\circ} \mathrm{C}$ as shown in Fig. 6. Thus, the $N_{e f f} M_{S}$ term in the micromagnetic model is less temperature dependent than $\alpha H_{A}$. Hence, the $N_{\text {eff }} M_{S}$ term will compensate for $\alpha H_{A}$ term at elevated temperature, thus the lower values of $N_{\text {eff }} M_{S}$ suppress the thermal degradation of the coercivity. The micromagnetic simulation results (Fig. 5) show that $N_{\text {eff }}$ decreases with decreasing grain size. Thus, the effect of the stray field is smaller at elevated temperature, explaining the better thermal stability of coercivity for the $\mathrm{Nd}$ $\mathrm{Fe}-\mathrm{B}$ permanent magnets with smaller grain size.

As shown in these examples, the experimentally observed grain size dependence of coercivity and grain size dependence of the thermal stability of coercivity in exchange coupled anisotropic Nd-Fe-B sintered magnets are qualitatively reproduced by micromagnetic simulations. The coercivity increase with decreasing grain size was found to be due to the reduction in the effective stray field arising from neighboring grains. The improved thermal stability of coercivity by the reduction of grain size is also attributed to the lower effective demagnetization constant, $N_{\text {eff }}$.

\section{Effect of Grain Shape on Coercivity of Nd-Fe-B Per- manent Magnets}

Grain shape is one of the microstructural parameters of permanent magnets, which can have a significant contribution to the coercivity. It is known numerically that different grain shape leads to different demagnetizing factors. Osborn has calculated the demagnetizing factors of general ellipsoid with different ellipsoid semi-axes ${ }^{32)}$. The origin of the change in the demagnetizing factors for different grain shapes originates from different surface charges. Thielsch et al. showed that change of aspect ratio of platelet shaped $\mathrm{Nd}_{2} \mathrm{Fe}_{14} \mathrm{~B}$ grains with a size and shape of comparable to that of hot-deformed $\mathrm{Nd}-\mathrm{Fe}-\mathrm{B}$ magnets, local demagnetizing field varies result in shape dependency of coercivity ${ }^{33)}$. Bance et al. reported that by increase of aspect ratio of prolate spheroids coercivity can

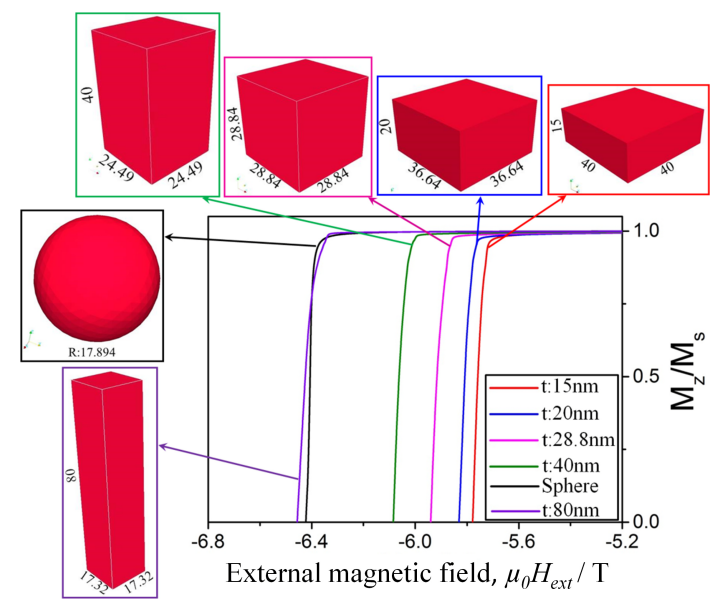

Fig. 7 Demagnetization curves for an $\mathrm{Nd}_{2} \mathrm{Fe}_{14} \mathrm{~B}$ grain with different shape and the same volume. Dimension scales are in nanometer.

be enhanced ${ }^{34)}$. Here, using micromagnetic simulations, the absolute demagnetization field prior to the nucleation and its influence to the coercivity for different grain shapes is calculated. Figure 7 shows demagnetization curves of single grains with the same volume but with different aspect ratios. These single grains can be correlated to the intrinsic coercivity of magnetically isolated $\mathrm{Nd}_{2} \mathrm{Fe}_{14} \mathrm{~B}$ grain inside of a bulk magnet. Note that in these models, no surface defect was considered. The smallest coercivity corresponds to the single platelet shaped grain with a height of $15 \mathrm{~nm}$. The coercivity value increases as the thickness of the cuboidal shaped grain increases. The largest coercivity corresponds to the $80 \mathrm{~nm}$ thick elongated grain which is slightly higher than that of spherical shaped $\mathrm{Nd}_{2} \mathrm{Fe}_{14} \mathrm{~B}$ grains.

Enhancement of coercivity by increase of the aspect ratio is in agreement with recently reported micromagnetic simulations by Bance et al. ${ }^{34)}$. Energy barrier calculations by Forster et al. on a columnar shaped ferromagnetic grain showed that by increase of column length, the required energy barrier for the magnetization reversal also increases which is beneficial for the enhancement of coercivity ${ }^{35)}$. To understand the effect of demagnetization field to the coercivity of a grain with different shapes, the demagnetization field right before the nucleation of a reverse domain was calculated. Note that the largest demagnetization field exists at a nucleation site, and the demagnetization field perpendicular to the easy axis applies the largest torque to tilt the magnetization vector out of the easy axis direction. Figure 8 shows the demagnetization field prior to the nucleation of the reversed domain, at the point that nucleation starts, for the $\mathrm{Nd}_{2} \mathrm{Fe}_{14} \mathrm{~B}$ grains with different shapes, i.e., a platelet-shape, a cuboid, a tetragonal shape and a sphere. The values of demagnetization field of the platelet shaped grain is the largest and this value decreases by increasing the aspect ratio of the grains from platelet to elongated cuboidal grain and the minimum value was found for the spherical shaped grain. This suggests that the non-uniform magnetization configuration at the corner of the platelet shaped $\mathrm{Nd}_{2} \mathrm{Fe}_{14} \mathrm{~B}$ grain is detrimental to the coercivity. However, with increase of the aspect ratio, the demagnetization field perpendicular to easy axis of the grain is reduced indicating that there is a weaker torque for tilting the magnetiza- 


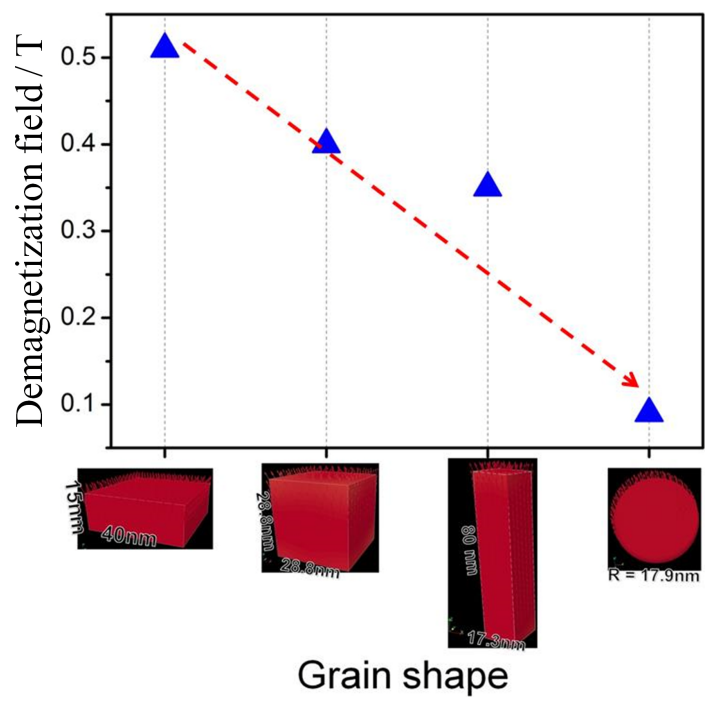

Fig. 8 Demagnetization field right before the nucleation field of a $\mathrm{Nd}_{2} \mathrm{Fe}_{14} \mathrm{~B}$ grain with platelet, cubic, elongated cuboidal, and spherical shaped grain. Note that the volume of all grains with different shape is the same.

a)

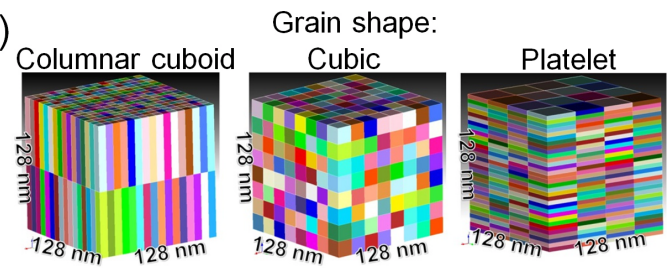

b)

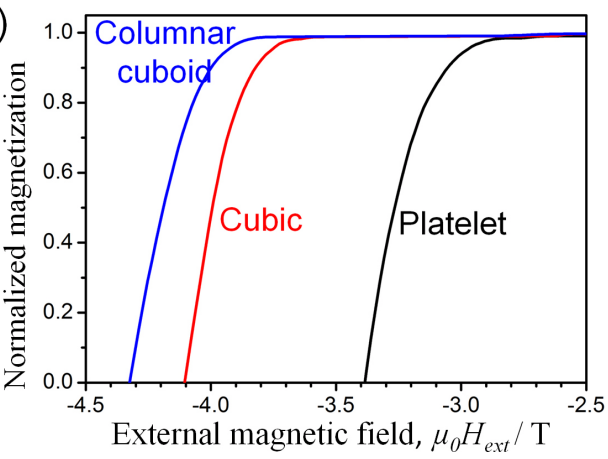

Fig. 9 (a) Modeled geometries with different $\mathrm{Nd}_{2} \mathrm{Fe}_{14} \mathrm{~B}$ grain shapes of platelet, cubic, and elongated cuboidal shape in a partially exchange coupled model. Note that the volume of all grains with different shape is the same. (b) Simulated demagnetization curves for the models with different grain shapes.

tion vector out of the easy axis direction that hinders the nucleation of the reversed magnetic domain.

The demagnetization field was calculated for a magnetically isolated single grain in the above. However, the question is how the demagnetization field influences the coercivity of an exchange coupled model with different grain shape. A cubic model with a size of $128 \times 128 \times 128 \mathrm{~nm}^{3}$ consisting of $\mathrm{Nd}_{2} \mathrm{Fe}_{14} \mathrm{~B}$ grains with different shapes of platelet, cubic, and elongated cuboid were modeled as shown in Fig. 9 (a). $2 \mathrm{~nm}$ thin amorphous grain boundary phase with $K_{1}=0 \mathrm{MJ} / \mathrm{m}^{3}$, $\mu_{0} M_{s}=0.5 \mathrm{~T}$ and $A=4 \mathrm{pJ} / \mathrm{m}$ was considered separating individual grains. By introducing this grain boundary phase, $\mathrm{Nd}_{2} \mathrm{Fe}_{14} \mathrm{~B}$ grains are partially exchange coupled while the grain boundary phase has three times smaller saturation mag- netization than the $\mathrm{Nd}_{2} \mathrm{Fe}_{14} \mathrm{~B}$ grains ${ }^{36)}$. Note that the volume of each $\mathrm{Nd}_{2} \mathrm{Fe}_{14} \mathrm{~B}$ grains was kept constant. Figure 9 (b) shows simulated demagnetization curves of these three models. It was found that the model with exchanged coupled $\mathrm{Nd}_{2} \mathrm{Fe}_{14} \mathrm{~B}$ grains with platelet-shaped grains shows lower coercivity and by change of the grain shape to cuboid and columnar cuboid, coercivity increases. This data indicate the minimum coercivity is expected for the platelet-shaped $\mathrm{Nd}_{2} \mathrm{Fe}_{14} \mathrm{~B}$ grain that might be due to a larger stray field. This is the case of the hot-deformed magnets. Using an analytical approach, Fukada et al. also reported a higher demagnetization factor for platelet shaped ferromagnetic grain ${ }^{37)}$. The coercivity can be enhanced by changing the shape of the grains to equiaxed or columnar grains, in which the stray field can be reduced.

\section{Effect of Grain Boundary Phase on Coercivity of Nd- Fe-B Magnets}

It is known that the coercivity of Nd-Fe-B based permanent magnets is strongly influenced by the structure and chemistry of the grain boundaries. Microstructure observations using transmission electron microscopy have revealed that $\mathrm{Nd}_{2} \mathrm{Fe}_{14} \mathrm{~B}$ grains in the Nd-Fe-B based permanent magnets are enveloped by a thin $(\sim 2-5 \mathrm{~nm})$ grain boundary phase ${ }^{1,13}$. Hence, depending on the magnetization of the grain boundary phase, i.e. ferromagnetic or non-ferromagnetic, the coercivity of magnets dramatically changes. The grain boundary phase is mainly composed of $\mathrm{Fe}, \mathrm{Nd}$ and $\mathrm{Cu}$ and its magnetization correlates to its chemical composition, i.e. the amount of Fe. Recent atom probe analyses have revealed that the grain boundary phase in $\mathrm{Nd}-\mathrm{Fe}-\mathrm{B}$ sintered magnets contain a substantial amount of $\mathrm{Fe}$ and $\mathrm{Co}, \sim 65$ at. $\%^{2)}$. Even higher amount of $\mathrm{Fe}$ and $\mathrm{Co}$ was reported in the intergranular phase of hot-deformed Nd-Fe-B magnets ${ }^{38)}$. The higher amount of $\mathrm{Fe}$ and $\mathrm{Co}$ in the intergranular phase of ultra-fine grain sized hot-deformed magnet was proposed to be the reason for their low coercivity. The highest coercivity of hot-deformed magnets is comparable to that of Nd-Fe-B sintered magnets that have an order of magnitude larger grain size as shown in Fig. 3 (b). Hence, a better understanding of the effect of the chemical composition of the intergranular phase in the hot-deformed magnets was strongly desired. There have been several experimental studies reporting the composition of the grain boundary phase and its magnetic behavior ${ }^{14,36,39)}$. However, tuning the magnetic properties of the grain boundary phase and studying its correlation to the coercivity is very challenging experimentally. Hence, micromagnetic simulations have been employed to study the influence of the grain boundary phase composition and its correlation to the magnetization reversals ${ }^{25,38,40-44)}$.

Figure 10 shows simulated demagnetization curves of a modeled hot-deformed magnet in which $\mathrm{Nd}_{2} \mathrm{Fe}_{14} \mathrm{~B}$ grains are separated with $4 \mathrm{~nm}$ thick grain boundary phase ${ }^{38)}$. The saturation magnetization $\left(\mu_{0} M_{\mathrm{s}}\right)$ and exchange stiffness $(A)$ of the grain boundary phase was varied from $1.2 \mathrm{~T}$ to $0.0 \mathrm{~T}$ and from $8 \mathrm{pJ} / \mathrm{m}$ to $0 \mathrm{pJ} / \mathrm{m}$. Coercivity and nucleation field are enhanced by the reduction of the saturation magnetization of the grain boundary phase. Snap shots of the magnetization configurations at the nucleation point for the models with 

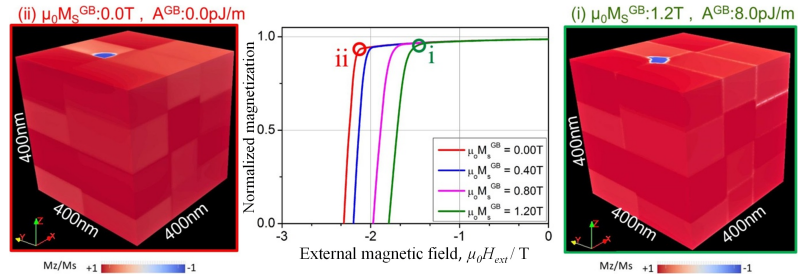

Fig. 10 Simulated demagnetization curves of modeled hot-deformed magnets in which $\mathrm{Nd}_{2} \mathrm{Fe}_{14} \mathrm{~B}$ grains are separated with $4 \mathrm{~nm}$ thick grain boundary phase. The saturation magnetization $\left(\mu_{0} \mathrm{M}_{\mathrm{s}}\right)$ and exchange stiffness of the grain boundary phase was varied between $1.2 \mathrm{~T}$ to $0.0 \mathrm{~T}$ and $8 \mathrm{pJ} / \mathrm{m}$ to $0 \mathrm{pJ} / \mathrm{m}$. The magnetization configuration of the modeled sample with GB magnetization of (i) $1.2 \mathrm{~T}$ and (ii) $0.0 \mathrm{~T}$ at nucleation fields. Reprinted with permission from Elsevier ${ }^{38)}$. a)
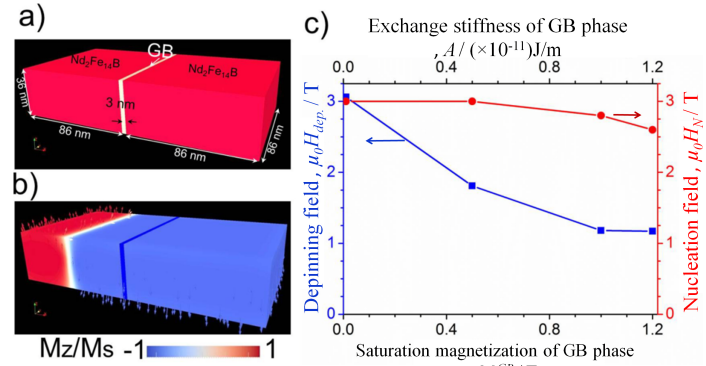

, $\mu_{0} M_{s}^{G B} / \mathrm{T}$
Fig. 11 (a) A modeled geometry including two $\mathrm{Nd}_{2} \mathrm{Fe}_{14} \mathrm{~B}$ grains separated with $3 \mathrm{~nm}$ thick intergranular phase. The surface of $\mathrm{Nd}_{2} \mathrm{Fe}_{14} \mathrm{~B}$ grains consist of $3 \mathrm{~nm}$ thick defect region with reduced magnetocrystalline anisotropy of $0 \mathrm{~kJ} / \mathrm{m}^{3}$ and exchange stiffness and magnetization comparable to $\mathrm{Nd}_{2} \mathrm{Fe}_{14} \mathrm{~B}$ grains. (b) Initial magnetization state of the model before applying magnetic field. Red and blue colors in (b) correspond to $\mathrm{Mz} / \mathrm{Ms}=$ +1 and -1 respectively. (c) Calculated depinning field and nucleation field versus the saturation magnetization and exchange stiffness of the intergranular phase.

non-ferromagnetic and ferromagnetic grain boundary phase are shown in Fig. 10. A non-ferromagnetic grain boundary phase prevents the propagation of a reversed domain into the neighboring grains. This simulation result suggests that the grain boundary phase is acting as pinning sites against the propagation of magnetic domain walls ${ }^{38)}$. The pinning strength of the grain boundary phase against magnetic domain wall motion is correlated with the intrinsic magnetic properties of the grain boundary phase, its thickness and domain wall width in the ferromagnetic grain. To calculate the change of the pinning strength at the grain boundary phase depending on its $\mu_{0} M_{s}$ and $A$, a simple micromagnetic simulation was carried out. Figure 11 shows two grain model separated by a $3 \mathrm{~nm}$ thick intergranular phase. The surface of the $\mathrm{Nd}_{2} \mathrm{Fe}_{14} \mathrm{~B}$ grains consist of $3 \mathrm{~nm}$ thick defect region with a reduced magnetocrystalline anisotropy of $0 \mathrm{~kJ} / \mathrm{m}^{3}$ and the saturation magnetization and exchange stiffness are similar to those of $\mathrm{Nd}_{2} \mathrm{Fe}_{14} \mathrm{~B}$. The initial magnetization state of the model is shown in Fig. 11 (b), in which one grain contains two domains and a Bloch type domain wall while the neighboring grain is in a single domain state. On applying external magnetic field, the domain wall moves to the right direction inside of the grain to reach to the intergranular phase, where it is pinned. The domain wall is depinned on the application of higher external field and the magnetization of the neighboring grain is switched. The required field to depin the do- main wall from the intergranular phase was measured as depinning field and was compared with the nucleation field of the model including the surface defect region. The saturation magnetization $\left(\mu_{0} M_{s}\right)$ of the intergranular phase was varied from $1.2 \mathrm{~T}$ to $0.0 \mathrm{~T}$ and exchange stiffness $(A)$ was decreased from $12 \mathrm{pJ} / \mathrm{m}$ to $0 \mathrm{pJ} / \mathrm{m}$. Figure 11 (c) compares the nucleation field and depinning field as a function of the magnetization and exchange stiffness of the intergranular phase. The depinning field of the intergranular phase increases with the reduction of the magnetization and exchange stiffness of the intergranular phase. However, the value of depinning field is far below the nucleation field in this model. The depinning field becomes as high as the nucleation field when the intergranular phase is non-ferromagnetic to exchange-decouples the $\mathrm{Nd}_{2} \mathrm{Fe}_{14} \mathrm{~B}$ grains. In fact, there is no depinning of the magnetic domain wall when a nonmagnetic grain boundary phase decouples two $\mathrm{Nd}_{2} \mathrm{Fe}_{14} \mathrm{~B}$ grains and magnetization reversal of neighboring grain also occurs by nucleation. This means that there is no distinction between the nucleation field and pinning field when ferromagnetic grains are exchange decoupled by the non-ferromagnetic grain boundary phase.

Figure 3 (b) shows that the coercivity of hot-deformed magnets is much lower than that expected from the extrapolated line of the grain size dependence of the coercivity of Nd-Fe-B sintered magnets. The 3DAP analyses by Liu et al. have shown that the $\mathrm{Nd}$ concentration in the intergranular phase in the hot-deformed magnets is substantially lower than that in the grain boundary phase in the Nd-Fe-B sintered magnets. This suggests that the modification of the chemical composition of the intergranular phase may lead to a higher coercivity in hot-deformed Nd-Fe-B magnets. Fukunaga et al. reported that the effect of exchange coupling in nano-sized grain magnets is pronounced compare to that of large grain sized magnets ${ }^{18)}$. More recently, Sepehri-Amin et al. studied the tendency of the coercivity depending on the magnetization of the intergranular phase using a micromagnetic model similar to the microstructure of hot-deformed Nd-Fe-B magnets as shown in Fig. 12 (a) and (b) for the grain sizes of 60 and $150 \mathrm{~nm}$ in the lateral direction ${ }^{13)}$. The $\mathrm{Nd}_{2} \mathrm{Fe}_{14} \mathrm{~B}$ grains are separated with a $3 \mathrm{~nm}$ thick intergranular layer in all the models. The saturation magnetization $\left(\mu_{0} M_{\mathrm{s}}\right)$ and the exchange stiffness $(A)$ of the intergranular phase was chosen as $1.2 \mathrm{~T}$ and $12 \mathrm{pJ} / \mathrm{m}$ for the model to simulate the exchange coupled $\mathrm{Nd}_{2} \mathrm{Fe}_{14} \mathrm{~B}$ grains. The saturation magnetization $\left(\mu_{0} M_{\mathrm{s}}\right)$ and exchange stiffness $(A)$ of the intergranular phase was varied as $\mu_{0} M_{\mathrm{s}}=1.2 \mathrm{~T}$ and $A=12 \mathrm{pJ} / \mathrm{m}$ and $\mu_{0} M_{\mathrm{s}}=$ $0.03 \mathrm{~T}$ and $A=1 \mathrm{pJ} / \mathrm{m}$ to simulate the effect of inter-grain exchange coupling. The simulated demagnetization curves for the models with grain sizes of 150 and $60 \mathrm{~nm}$ are shown in Fig. 12 (c). The exchange coupled model with grain sizes of $150 \mathrm{~nm}$ and $60 \mathrm{~nm}$ show the same coercivity of $3.19 \mathrm{~T}$. However, the coercivity of the weakly exchange coupled model with the grain size of $60 \mathrm{~nm}$ is increased to $4.05 \mathrm{~T}$. This result indicates that the role of the intergranular phase is more pronounced in the hot-deformed $\mathrm{Nd}-\mathrm{Fe}-\mathrm{B}$ magnets, i.e., minimizing the ferromagnetic exchange coupling is more effective to achieve a high coercivity in fine grained magnets

In order to reduce the magnetization of the grain boundary phase, low temperature eutectic diffusion process using $\mathrm{Nd}_{70} \mathrm{Cu}_{30}$ alloy powders has been introduced ${ }^{42,43)}$, by which 
a)

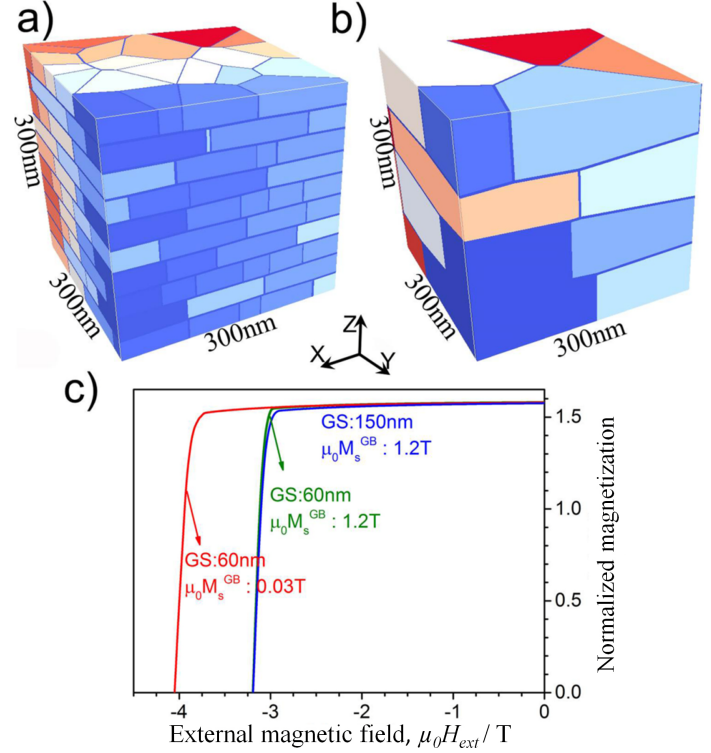

Fig. 12 Modeled hot-deformed Nd-Fe-B magnets with average grain size in lateral direction of (a) $60 \mathrm{~nm}$, and (b) $150 \mathrm{~nm}$. (c) Demagnetization curves of simulated hot-deformed Nd-Fe-B magnets with the average grain size in lateral direction of $150 \mathrm{~nm}$ and $60 \mathrm{~nm}$. The saturation magnetization of intergranular phase $\left(\mu_{0} \mathrm{M}_{\mathrm{s}}^{\mathrm{GB}}\right)$ was chosen as $1.2 \mathrm{~T}$ for $150 \mathrm{~nm}$ grain sized model while it was $1.2 \mathrm{~T}$ and $0.03 \mathrm{~T}$ for $60 \mathrm{~nm}$ grain sized model. Reprinted with permission from Elsevier ${ }^{13)}$.

the coercivity of hot-deformed magnets was substantially enhanced. Microstructure characterizations of the diffusion processed hot-deformed Nd-Fe-B magnet has shown that the Fe and Co content of the grain boundary phase can be substantially reduced, resulting in the enhancement of coercivity in the hot-deformed magnets as high as $2.3 \mathrm{~T}$. Detailed microstructure characterizations using SEM, TEM and 3DAP have shown that the intergranular phases located perpendicular to the easy axis of $\mathrm{Nd}_{2} \mathrm{Fe}_{14} \mathrm{~B}$ grains are free from $\mathrm{Fe}$ and $\mathrm{Co}$, while the intergranular phase parallel to the c-axis of $\mathrm{Nd}_{2} \mathrm{Fe}_{14} \mathrm{~B}$ grains still contain large amount of $\mathrm{Fe}$ and $\mathrm{Co}$. This suggests that the intergranular phase on the side of the platelet-shaped $\mathrm{Nd}_{2} \mathrm{Fe}_{14} \mathrm{~B}$ grains is ferromagnetic. Figure 13 shows micromagnetic simulation results on how the side grain boundary phase influences the magnetization reversal of hot-deformed magnets. Figure 13 (a) shows a modeled hot-deformed magnet with a size of $1 \times 1 \times 1 \mu \mathrm{m}^{3}$. Demagnetization curves of two models were simulated as shown in Fig. 13 (b): (i) the side grain boundary phase is ferromagnetic with $\mu_{0} M_{s}=0.5 \mathrm{~T}$ and $A=8 \mathrm{pJ} / \mathrm{m}$ and (ii) the side grain boundary phase is non-ferromagnetic with $\mu_{0} M_{s}=0.0 \mathrm{~T}$ and $A=0 \mathrm{pJ} / \mathrm{m}$. Note that in both cases, the grain boundary phase parallel to c-plane is assumed to be non-ferromagnetic. This simulation result shows that the reduction of the magnetization of the side grain boundary phase in hot-deformed $\mathrm{Nd}$ $\mathrm{Fe}-\mathrm{B}$ magnets leads to further enhancement of coercivity. Snap shots of the magnetization configurations at the nucleation point for the models with ferromagnetic and non-ferromagnetic side grain boundaries are shown in Fig. 13 (c) and (d). This result indicates that the magnetization reversal starts at the side ferromagnetic grain boundary phase at a lower nucleation field, the propagation of which is hindered by introducing a non-ferromagnetic phase at the side grain boundar-
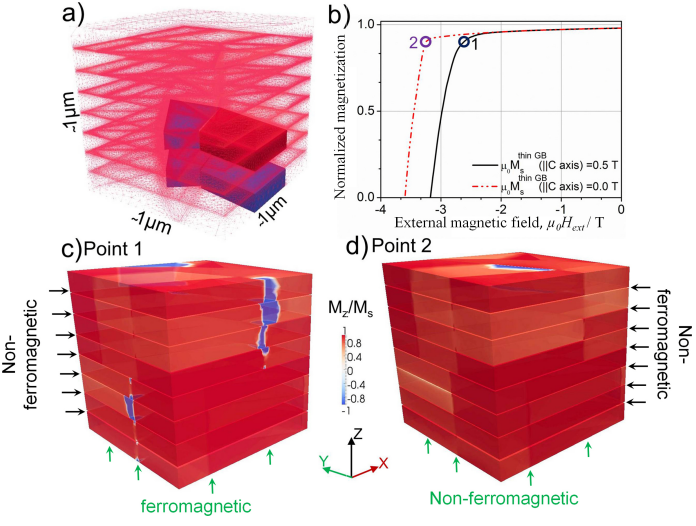

Fig. 13 (a) The modeled hot-deformed magnet with a size of $1 \mu \mathrm{m}^{3}$ showing the cuboids of $\mathrm{Nd}_{2} \mathrm{Fe}_{14} \mathrm{~B}$ grains with the applied tetrahedron meshes. (b) Demagnetization curves of the simulated samples with different magnetization for thin intergranular phase $(0 \mathrm{~T}$ and $0.5 \mathrm{~T})$ located parallel to c-axis of $\mathrm{Nd}_{2} \mathrm{Fe}_{14} \mathrm{~B}$ grains. The snap shots of magnetization configuration at nucleation point of the modeled samples with thin GB saturation magnetizations of (c) $0.5 \mathrm{~T}$ and (d) $0.0 \mathrm{~T}$. Reprinted with permission from Elsevier $^{43}$ )

ies. More recently, micromagnetic simulations of polycrystalline anisotropic Nd-Fe-B magnets by Fujisaki et al. showed that reduction of saturation magnetization and exchange stiffness of grain boundaries located parallel to c-axis of $\mathrm{Nd}_{2} \mathrm{Fe}_{14} \mathrm{~B}$ grains enhances the coercivity of Nd-Fe-B magnets more efficiently than that parallel to c-plane ${ }^{40)}$. Based on these results, reduction of the magnetization and exchange stiffness of the grain boundaries located to parallel to c-axis of $\mathrm{Nd}_{2} \mathrm{Fe}_{14} \mathrm{~B}$ grains in the hot-deformed $\mathrm{Nd}-\mathrm{Fe}-\mathrm{B}$ magnets as well as Nd-Fe-B sintered magnets can lead to the further enhancement of coercivity.

\section{Discussion and Future Directions}

Recent investigations have shown that the upper limit of coercivity of hot-deformed magnets by the grain boundary modification using the eutectic diffusion process is about 2.3 $\mathrm{T}$ for $\mathrm{Nd}-\mathrm{Cu}$ and $2.5 \mathrm{~T}$ for $\mathrm{Nd}-\mathrm{Al}$. However, the hot-deformed magnets diffusion processed with $\mathrm{Nd}-\mathrm{Al}$ does not exhibit good thermal stability of coercivity because of the partial dissolution of $\mathrm{Al}$ into the $\mathrm{Nd}_{2} \mathrm{Fe}_{14} \mathrm{~B}$ lattice. Further enhancement of coercivity of the hot-deformed magnets, in particular at elevated temperature, can be achieved by forming thin Dy-rich shell in the $\mathrm{Nd}_{2} \mathrm{Fe}_{14} \mathrm{~B}$ grains. Figure 14 (a) shows a modeled hot-deformed magnet with a size of $1 \mu \mathrm{m} \times$ $1 \mu \mathrm{m} \times 800 \mathrm{~nm}$. In this model, $\mathrm{Nd}_{2} \mathrm{Fe}_{14} \mathrm{~B}$ grains are enveloped with $5 \mathrm{~nm}$ thick intergranular phase. The magnetization of the intergranular phase was varied from $0.0 \mathrm{~T}$ to $0.5 \mathrm{~T}$. The shell phase with a thickness of $7 \mathrm{~nm}$ was taken into account at the outer region of $\mathrm{Nd}_{2} \mathrm{Fe}_{14} \mathrm{~B}$ grains as shown in Fig. 14 (b). Two conditions were assumed for the shell region as $\mathrm{Nd}_{2} \mathrm{Fe}_{14} \mathrm{~B}$ or $\left(\mathrm{Nd}_{0.9}, \mathrm{Dy}_{0.1}\right)_{2} \mathrm{Fe}_{14} \mathrm{~B}$ phases. The demagnetization curves of the models with different magnetizations of the thin intergranular phase and with or without Dy-rich shell layers are shown in Fig. 14 (c). For ferromagnetic grain boundary phase, coercivity is improved by introducing the $\left(\mathrm{Nd}_{0.9} \mathrm{Dy}_{0.1}\right)_{2} \mathrm{Fe}_{14} \mathrm{~B}$ shell. However, the modification of the grain boundary phase to a non-ferromagnetic phase leads to a 

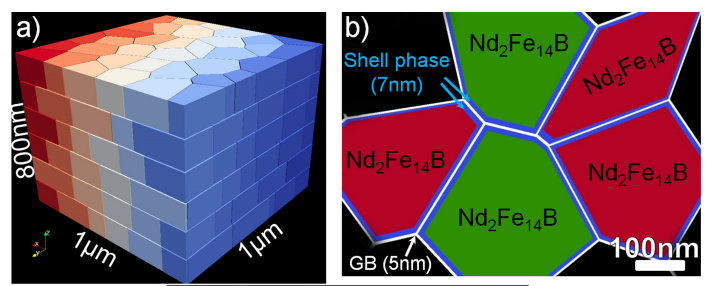

c)

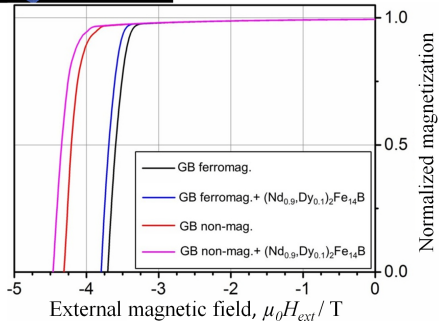

Fig. 14 (a) Modeled hot-deformed magnet with a size of $1 \times 1 \times 0.8 \mu \mathrm{m}^{3}$. (b) The schematic image of the microstructure of the modeled hot-deformed magnet. (c) The demagnetization curves of the simulated samples with different magnetization $(0$ and $0.5 \mathrm{~T})$ of the grain boundary phase and with and without Dy-rich shell phase.
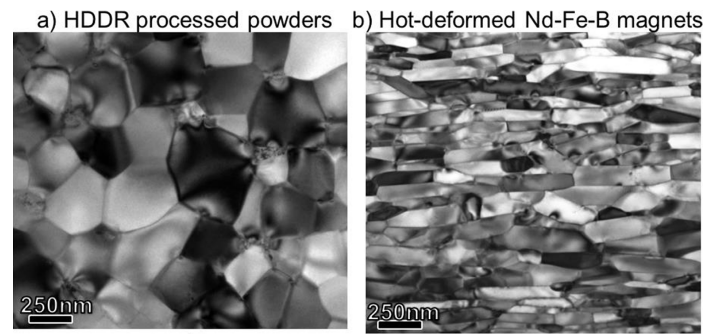

Fig. 15 Bright field TEM images obtained from (a) HDDR processed Nd$\mathrm{Fe}-\mathrm{B}$ powders and (b) hot-deformed Nd-Fe-B magnets.

further enhancement of coercivity. The highest coercivity is achieved in the model with non-ferromagnetic grain boundary layer and a thin $\left(\mathrm{Nd}_{0.9} \mathrm{Dy}_{0.1}\right)_{2} \mathrm{Fe}_{14} \mathrm{~B}$ shell. Sepehri-Amin et al. demonstrated a similar microstructure in hot-deformed magnets by applying the grain boundary diffusion process using a $\mathrm{Nd}_{60} \mathrm{Dy}_{20} \mathrm{Cu}_{20}$ eutectic alloy to the hot-deformed $\mathrm{Nd}$ Fe-B magnets, leading to a high coercivity of $2.6 \mathrm{~T}^{44)}$. However, this high coercivity hot-deformed magnet showed a very small remanent magnetization of $1.10 \mathrm{~T}$ due to the large volume fraction of the intergranular phase. Further work is necessary to reduce the thickness of the intergranular phase to minimize the reduction of the magnetization.

Micromagnetic simulations showed smaller grain size is beneficial for achieving a higher coercivity and a better thermal stability of coercivity. In addition, based on micromagnetic simulation results, coercivity of hot-deformed Nd-Fe-B magnets with platelet-shape grains are expected to be lower than those of equiaxed grain structure for the same grain size. Figure 15 show typical microstructures of hot-deformed Nd$\mathrm{Fe}-\mathrm{B}$ magnets and hydrogen disproportion desorption recombination (HDDR) processed Nd-Fe-B magnets. Although both show ultrafine grain size comparable to the single domain particles size $\left(\sim 250 \mathrm{~nm}\right.$ for $\left.\mathrm{Nd}_{2} \mathrm{Fe}_{14} \mathrm{~B}\right)$, the HDDR magnets are expected to show higher coercivity when the magnetic isolation is achieved because of the lower demagnetization field expected for the equiaxed grain structure.

It is known that for a realistic calculation of nucleation field in modeled Nd-Fe-B magnets, the mesh size has to be smaller than the exchange length of $\mathrm{Nd}_{2} \mathrm{Fe}_{14} \mathrm{~B}$ phase, $\delta=$ $(A / K)^{1 / 2}=1.7 \mathrm{~nm}$, in which $A$ is exchange length and $K$ is magnetocrystalline anisotropy constant of $\mathrm{Nd}_{2} \mathrm{Fe}_{14} \mathrm{~B}$ phase ${ }^{3)}$. Considering the grain size range of $\mathrm{Nd}-\mathrm{Fe}-\mathrm{B}$ magnets, $\sim 250 \mathrm{~nm}$ for HDDR processed magnets and hot-deformed magnets to $\sim 5 \mu \mathrm{m}$ for $\mathrm{Nd}-\mathrm{Fe}-\mathrm{B}$ sintered magnets, realistic micromagnetic simulation of Nd-Fe-B magnets needs computation of large scaled models. This is the current challenge for conventional micromagnetic simulations to simulate large grained materials. Hence, development of a new algorithm that can speed up the calculation time is necessary enabling us to simulate large scaled models. Recently, Exl et al. has introduced a steepest decent energy minimization method for micromagnetics to simulate quasistatic hysteresis loops ${ }^{45}$. Exl et al. reported that this new method is faster than a Landau-Lifshitz micromagnetic solver by more than a factor of two ${ }^{45)}$. The steepest descent energy minimization method was also implemented on a graphic processor unit (GPU) resulted in 4.8 times faster calculation time compared to that of single-core central processing unit $(\mathrm{CPU})^{45)}$. Using the same approach, Furuya et al. also reported a successful simulation of a permanent magnet including 20.7 million elements ${ }^{46)}$. These efforts indicate that using steepest decent energy minimization technique implemented for micromagnetic, hysteresis loop simulation of large sized models is feasible which is necessary for realistic simulation of large grain sized permanent magnets.

\section{Summary}

Micromagnetic simulations reproduce the grain size dependence of Nd-Fe-B magnets, which is attributed to the decrease of local demagnetization factor for finer grain size. Lower demagnetization factor leads to improved temperature dependence of coercivity, hence the fine grain sized magnets like hot-deformed magnets show improved temperature dependence of coercivity compared to sintered magnets. In order to gain the benefits from the small grain size, $\mathrm{Nd}_{2} \mathrm{Fe}_{14} \mathrm{~B}$ grains must be isolated magnetically by introducing non-ferromagnetic grain boundary phase. Grain morphology also gives large influence on the coercivity because demagnetization field strongly depends on the grain morphology. Therefore, HDDR processed $\mathrm{Nd}-\mathrm{Fe}-\mathrm{B}$ magnets with equiaxed grain morphology have a better potential to attain a higher coercivity than that of hot-deformed Nd-Fe-B magnets with platelet-shaped grains.

\section{Acknowledgment}

This work was in part supported by CREST, JST.

\section{REFERENCES}

1) K. Hono and H. Sepehri-Amin: Scr. Mater. 67 (2012) 530.

2) H. Sepehri-Amin, T. Ohkubo, T. Shima and K. Hono: Acta Mater. 60 (2012) 819.

3) W. Rave, K. Ramstöck and A. Hubert: J. Magn. Magn. Mater. 183 (1998) 329.

4) Jr W. F. Brown: Micromagnetics, (Interscience Publishers, New York 1963) p. 68 
5) D. Kinderlehrer and L. Ma: IEEE Trans. Magn. 30 (1994) 4380.

6) T. Schrefl, G. Hrkac, S. Bance, D. Suess, O. Ertl, J. Fidler, in Handbook of Magnetism and Advanced Magnetic Materials, edited by H. Kronmüller and S. Parkin, (John Wiley and Sons, 2007), Vol 2. pp. 765794.

7) T.L. Gilbert: IEEE Trans. Magn. 40 (2004) 3443.

8) R. Ramesh and K. Srikrishna: J. Appl. Phys. 64 (1988) 6406.

9) P. Nothnagel, K.H. Müller, D. Eckert and A. Handstein: J. Magn. Magn. Mater. 101 (1991) 379.

10) K. Uestuener, M. Katter and W. Rodewald: IEEE Trans. Magn. 42 (2006) 2897.

11) W.F. Li, T. Ohkubo, K. Hono and M. Sagawa: J. Magn. Magn. Mater. 321 (2009) 1100.

12) Y. Une, and M.Sagawa, Presented at REPM'10, Bled, Slovenia, 2010.

13) J. Liu, H. Sepehri-Amin, T. Ohkubo, K. Hioki, A. Hattori, T. Schrefl and K. Hono: Acta Mater. 82 (2015) 336.

14) Y. Murakami, T. Tanigaki, T.T. Sasaki, Y. Takeno, H.S. Park, T. Matsuda, T. Ohkubo, K. Hono and D. Shindo: Acta Mater. 71 (2014) 370.

15) T. Kohashi, K. Motai, T. Nishiuchi and S. Hirosawa: Appl. Phys. Lett. 104 (2014) 232408/1-232408/5.

16) H. Kronmüller, R. Fischer, R. Hertel and T. Leineweber: J. Magn. Magn. Mater. 175 (1997) 177.

17) W. Rave and K. Ramstöck: J. Magn. Magn. Mater. 171 (1997) 69.

18) H. Fukunaga and H. Inoue: Jpn. J. Appl. Phys. 31 (1992) 1347-1352.

19) T. Schrefl, H.F. Schmidts, J. Fidler and H. Kronmüller: IEEE Trans. Magn. 29 (1993) 2878.

20) H.F. Schmidts and H. Kronmüller: J. Magn. Magn. Mater. 94 (1991) 220.

21) J. Fidler and T. Schrefl: J. Appl. Phys. 79 (1996) 5029.

22) S. Bance, B. Seebacher, T. Schrefl, L. Exl, M. Winklhofer, G. Hrkac, G. Zimanyi, T. Shoji, M. Yano, N. Sakuma, M. Ito, A. Kato and A. Manabe: J. Appl. Phys. 116 (2014) 233903.

23) H. Sepehri-Amin, T. Ohkubo, M. Gruber, T. Schrefl and K. Hono: Scr. Mater. 89 (2014) 29.

24) H. Sepehri-Amin, Y. Une, T. Ohkubo, K. Hono and M. Sagawa: Scr. Mater. 65 (2011) 396.

25) J. Fujisaki, A. Furuya, Y. Uehara, K. Shimizu, H. Oshima, T. Ohkubo, S. Hirosawa and K. Hono: IEEE Trans. Magn. 50 (2014) 7100704.

26) S. Bance, J. Fischbacher, and T. Schrefl: J. Appl. Phys. 117 (2015) 17A733.

27) S. Bance and J. Fischbacher, A. kovacs, H. Oezelt, F. Reichel and T.
Schrefl: J. o. M. 67 (2015) 1350

28) S. Hirosawa, K. Tokuhara and M. Sagawa: Jpn. J. Appl. Phys. 26 (1987) L1359.

29) K.-D. Durst and H. Kronmüller: J. Magn. Magn. Mater. 68 (1987) 63.

30) S. Hock, 1988, PhD thesis, University of Stuttgart, Germany.

31) S. Hirosawa, Y. Matsuura, H. Yamamoto, S. Fujimura, M. Sagawa and H. Yamauchi: J. Appl. Phys. 59 (1986) 873.

32) J.A. Osborn: Phys. Rev. 67 (1945) 351

33) J. Thielsch, D. Suess, L. Schultz and O. Gutfleisch: J. Appl. Phys. 114 (2013) 223909.

34) S. Bance, J. Fischbacher, T. Schrefl, I. Zins, G. Rieger and C. Cassignol: J. Magn. Magn. Mater. 363 (2014) 121.

35) H. Forster, N. Bertram, X. Wang, R. Dittrich and T. Schrefl: J. Magn. Magn. Mater. 267 (2003) 69.

36) H. Sepehri-Amin, T. Ohkubo, T. Shima and K. Hono: Acta Mater. 60 (2012) 819.

37) T. Fukada, M. Matsuura, R. Goto, N. Tezuka, S. Sugimoto, Y. Une and M. Sagawa: Mater. Trans. 53 (2012) 1967.

38) J. Liu, H. Sepehri-Amin, T. Ohkubo, K. Hioki, A. Hattori, T. Schrefl and K. Hono: Acta Mater. 61 (2013) 5387.

39) T. Kohashi, K. Motai, T. Nishiuchi and S. Hirosawa: Appl. Phys. Lett. 104 (2014) 232408.

40) J. Fujisaki, A Furuya, Y. Uehara, K. Shimizu, T. Ataka, T. Tanaka, H. Oshima, T. Ohkubo, S. Hirosawa and K. Hono: Presented at $13^{\text {th }}$ joint MMM-Intermag conference, San Diego, (2016).

41) T. Oikawa, H. Yokota, T. Ohkubo and K. Hono: AIP Advances 6 (2016) 056006.

42) H. Sepehri-Amin, T. Ohkubo, T. Nishiuchi, S. Hirosaw and K. Hono: Scr. Mater. 63 (2010) 1124.

43) H. Sepehri-Amin, T. Ohkubo, S. Nagashima, M. Yano, T. Shoji, A. Kato, T. Schrefl and K. Hono: Acta Mater. 61 (2013) 6622.

44) H. Sepehri-Amin, J. Liu, T. Ohkubo, K. Hioki, A. Hattori, T. Schrefl and K. Hono: Scr. Mater. 69 (2013) 647.

45) L. Exl, S. Bance, F. Reichel, T. Schrefl, H.P. Stimming, and N.J. Mauser: J. Appl. Phys. 115 (2014) 17D118.

46) A. Furuya, J. Fujisaki, K. Shimizu, Y. Uehara, T. Ataka, T. Tanaka and H. Oshima: IEEE Trans. Magn. 51 (2015) 2103004

47) N.M. Dempsey, T.G. Woodcock, H. Sepehri-Amin, Y. Zhang, H. Kennedy, D. Givord, K. Hono and O. Gutfleisch: Acta Mater. 61 (2013) 4920 . 